\title{
O pacote Shiny na criação de applets para o ensino de Funções Matemáticas e Estatística Descritiva na Educação Básica
}

Paula Ribeiro dos Santos (D)

\author{
Izabela Regina Cardoso de Oliveira(D) \\ Corbalan Góis FreireiD
}

Evelise Roman

\section{Resumo}

O uso de applets no processo de ensino-aprendizagem é uma forma de trazer os avanços tecnológicos para o ambiente educacional. Dentre diversas vantagens, isso pode promover mais dinamismo durante as aulas e contribuir para o desenvolvimento cognitivo do aluno. Nesse trabalho ilustramos o uso do pacote Shiny para a criação de aplicativos que podem ser usados no ensino de Funções Matemáticas e Estatística Descritiva. Os aplicativos foram implementados e podem ser acessados por professores e estudantes usando computadores, smartphones e tablets, dentro ou fora do ambiente de sala de aula. Além disso, os códigos e tutoriais são disponibilizados no site http://shiny.rstudio.com/tutorial/, para que professores sintam-se encorajados a modificarem e/ou criarem seus próprios aplicativos, proporcionando um ambiente de ensino mais lúdico e interativo.

Palavras-chave: aplicativos; educação matemática; R; RStudio.

\begin{abstract}
Currently we are facing with fast technological advances and the use of applets in the teachinglearning process is a way to bring them to the educational environment. Among several advantages, this can promote dynamism during classes and contribute to the student's cognitive development. In this work we use the Shiny $\mathrm{R}$ package while creating apps for teaching mathematical functions and descriptive statistics. Applications are presented and can be accessed by teachers and students through computers, smartphones and tablets, in or out classroom. Furthermore, codes are made available at http://shiny.rstudio.com/tutorial/, so teachers can modify and/or create their own applications, providing a more playful and interactive teaching environment.
\end{abstract}

Keywords: apps; Mathematic teaching; R; RStudio

\section{Introdução}

Matemática e Estatística são disciplinas que desempenham um papel fundamental na sociedade e, na maioria dos países, são introduzidas na Educação Básica. O uso de applets tem sido visto como uma maneira inovadora de aprimorar o ensino desses conteúdos ao promover dinamismo durante as aulas e torná-las mais interativas, colaborando para o desenvolvimento cognitivo do 
estudante [14]. Assim, é importante que os educadores conheçam e tenham acesso a ferramentas modernas que lhes permitam usufruir da tecnologia que está ao seu dispor durante o processo de ensino-aprendizagem.

O R é um programa livre, baseado na linguagem S e muito utilizado por estatísticos. No entanto, seu uso como ferramenta de ensino não se restringe a conceitos de Estatística [11]. Esse programa pode ser utilizado na sua forma original ou em uma dentre as várias interfaces gráficas disponíveis, como o RStudio. Esse é um ambiente de desenvolvimento integrado (IDE), com uma interface gráfica que possui grande interatividade com o usuário. Dentre suas várias funcionalidades, tem-se o pacote Shiny, que possibilita a criação de aplicativos interativos (Shiny apps) [2] [7].

A grande vantagem da criação de applets usando o Shiny é que isso não requer o conhecimento de uma linguagem de programação avançada como Java, JavaScript, HTML, o que desencorajaria muitos usuários a criar seus próprios aplicativos. O único conhecimento requerido é sobre a linguagem de programação R. Além disso, os aplicativos criados podem ser publicados e distribuídos em formato HTML e, para utilizá-los, não é necessário que o usuário (estudante, por exemplo) tenha o programa $\mathrm{R}$ instalado.

O pacote Shiny é relativamente recente, mas já encontra diversas aplicações nos ambientes científico, empresarial e educacional. Nesse último caso destaca-se sua utilização para o ensino de conceitos de Estatística em nível superior [8], [18] [9] [12]. No entanto são encontrados poucos relatos sobre o uso dessa ferramenta em outras áreas da educação [13], e, até o momento, nenhum direcionado à Educação Básica.

Assim, o principal objetivo deste estudo foi criar, implementar e disponibilizar aplicativos para o ensino de conceitos de Matemática e Estatística na Educação Básica. Assim, são apresentados recursos para diversificação didática com possibilidades de mudanças das aulas de Matemática e Estatística, privilegiando metodologias ativas para educação básica e superior. Foram abordados os conceitos de Funções Elementares e Estatística Descritiva, com interpretação de gráficos e conceitos de medidas de posição. Os aplicativos implementados foram disponibilizados on-line, o que possibilita que qualquer professor possa utilizá-los. Além disso, esses podem ser acessados por estudantes por meio de computadores, tablets e smartphones, a qualquer hora e em qualquer lugar, possibilitando um processo de aprendizado continuado, além da sala de aula.

\section{A resolução de problemas e o uso de aplicativos interativos em sala de aula (Mobile Learning)}

Frequentemente nos deparamos com situações em que há a necessidade da tomada de decisão. Muitas vezes essas situações indicam um problema que demanda pensamento e reflexão para sua solução. A busca dessa "implica refletir sobre como obter o fim desejado e por qual caminho". Quando não sabemos o caminho ou quando esse não é alcançável de imediato, isso provoca a mente até a chegada de uma solução mais apropriada ou correta [3].

A resolução de problemas é peça central no ensino de Matemática e há meios que podem facilitar essa prática, como as novas tecnologias de informação e comunicação (nTICs). Essas tornam o aluno agente ativo na construção do conhecimento, estimulando o raciocínio, criatividade, autonomia, tomada de decisão, autoconfiança e o prazer pela descoberta. As nTICs auxiliam o aluno a obter um melhor entendimento por meio da experimentação e visualização, de maneira mais dinâmica e rápida.

As principais nTICs que podem ser usadas na resolução de problemas são jogos digitais e softwares educativos de matemática. Softwares favorecem a aprendizagem por facilitarem a visualização de 
gráficos e sua interpretação, sendo necessário que o aluno associe seu conhecimento às informações fornecidas pelo programa.

Com a disseminação do uso de aparelhos sem fio, o uso de recursos de tecnologias em sala de aula deixou de se limitar ao acesso ao computador. A aprendizagem por meio de dispositivos móveis (M-Learning, do inglês Mobile Learning) inclui todas as tecnologias sem fio e computação móvel, contribuindo para que a aprendizagem possa ocorrer em qualquer tempo, lugar e espaço, dando autonomia para o aluno e dinamismo para o processo [10]. Aparelhos smartphones e tablets tornaram-se comuns no dia a dia do estudante. Dentro desse contexto, é preciso repensar a forma de uso desses aparelhos como ferramenta auxiliar no processo de ensino aprendizagem e, consequentemente, no uso de aplicativos. Esse novo contexto proporciona, também, um novo desafio para a formação docente.

Apesar da disseminação dos dispositivos móveis e da grande quantidade de aplicativos disponíveis para serem utilizados com foco de ensino, Borba et al. (2016) mostram, através de uma revisão completa da literatura, que os estudos sobre o uso de mobile learning no ensino de matemática ainda são escassos [4]. Consequentemente, ainda faltam propostas que abordem as tecnologias móveis como recursos de aprendizagem matemática na formação básica dos futuros professores $[10]$.

\subsection{O R e o RStudio}

Criado no início da década de 90, o R é um programa livre que permite o processamento de dados, cálculo, visualização gráfica, além de fornecer uma ampla variedade de técnicas estatísticas. Ele é disponível sobre os termos da "Licença Pública Geral do GNU" da Fundação do Software Livre (Free Software Foundation GNU General Public License) na forma de código fonte e é executável em diversas plataformas, incluindo Linux, Windows e MacOS. O R possui uma grande comunidade de usuários no mundo inteiro que colaboram com o aprimoramento e extensão do programa por meio de pacotes, que são bibliotecas para funções específicas. Há em torno de 25 pacotes embutidos na versão básica do R e muitos outros (cerca de 12000 pacotes) (R CORE TEAM, 2018) disponíveis para instalação. A versão mais recente do $\mathrm{R}$ está disponível para download [15].

O RStudio, definido como um IDE, é uma interface gráfica mais completa e intuitiva do R. Segundo Verzani (2011) o R, como outras linguagens de programação, é estendido através de funções escritas e um IDE é projetado para facilitar esse processo, incluindo um meio para a emissão de comandos de forma interativa.

A versão desktop do programa RStudio também está disponível para as principais plataformas. Seu download pode ser feito em http://www.rstudio.org/download/ e para executá-lo é necessário ter o programa $\mathrm{R}$ instalado.

\subsection{O pacote Shiny do RStudio}

O Shiny é um pacote criado pelos desenvolvedores do RStudio, que permite a fácil interface entre o R e um navegador web. É uma estrutura que facilita a construção de aplicativos interativos com o R. Uma de suas principais vantagens é que não há necessidade de combinar código $\mathrm{R}$ com um código JavaScript ou HTML, já que o mesmo contém características acopladas que cobrem as funcionalidades mais usadas em um aplicativo interativo. Basta que o usuário tenha conhecimento da linguagem $\mathrm{R}$ para criar seus aplicativos. 
A estrutura do aplicativo tem dois componentes: um script de interface de usuário (ui.R) e um script de servidor (server.R). O script ui.R controla o layout e a aparência do aplicativo, e o server.R contém as instruções que o computador precisa para construí-lo. Para cada aplicativo, a estrutura mínima são os scripts ui.R e server.R salvos em um mesmo diretório. Para mais detalhes sobre esses scripts, o leitor interessado pode consultar os tutoriais e manuais disponíveis em http://shiny.rstudio.com/tutorial/.

Os aplicativos Shiny são fáceis de serem compartilhados e a melhor maneira de fazer isso é por meio da própria URL, o que permite o acesso ao aplicativo por meio da internet com um navegador web.

\section{Metodologia}

Neste estudo, foram criados aplicativos voltados para o ensino dos conceitos de funções elementares e estatística descritiva na educação básica. Para isso foi utilizada a versão 3.4.2 do R, versão 1.1.414 do RStudio e o pacote Shiny. Todos os aplicativos foram publicados na plataforma shinyapps.io e podem ser acessados gratuitamente. O aplicativo para o estudo de funções matemáticas está disponível em https://appsensinobasico.shinyapps.io/funcoes/; o aplicativo de gráficos para variáveis qualitativas em https://appsensinobasico.shinyapps.io/qualitativo/; e o aplicativo de gráfico e medidas estatísticas de variáveis quantitativas em https://appsensinobasico.shinyapps.io/quantitativo/. Cada aplicativo criado vem acompanhado de uma instrução (Leia-me) e dos códigos (ui.R e server.R) utilizados na programação. Assim, os usuários interessados podem aprimorá-los, modificálos e/ou usá-los como base para a criação de seus próprios aplicativos. A seguir, são apresentados os aplicativos criados, juntamente com orientações para sua utilização no ambiente de sala de aula.

\section{Resultados}

\subsection{Aplicativos para o ensino de funções elementares matemáticas}

Nesta subseção é apresentado o aplicativo criado para o ensino de Funções Matemáticas na Educação Básica. De acordo com a BNCC, as funções de primeiro e segundo grau e funções exponenciais são trabalhadas no $1^{0}$ ano do Ensino Médio. A função de primeiro grau também é trabalhada no segundo ano do Ensino Médio em outra abordagem, junto com a função logarítmica. Além disso, a BNCC recomenda que seja desenvolvida a habilidade de reconhecer os efeitos de transição ou mudança de escala no gráfico de uma função.

Schreiber e Battisti (2017) afirmam que o conceito de função de $1^{o}$ grau é de difícil compreensão por grande parte dos alunos e destacam o quão importante é o conhecimento do assunto pelo professor e seu preparo antes de apresentar o tema [16]. A dificuldade de transmitir e compreender o assunto aumenta com a complexidade da função em estudo. Então, acreditamos que ferramentas que permitem a visualização gráfica de forma interativa podem contribuir para o processo de ensino-aprendizagem do tema.

O aplicativo Shiny criado, que é detalhado na sequência, engloba cinco funções (Tabela 1). Primeiramente o usuário pode escolher uma função dentre as disponíveis. Em seguida, os valores dos coeficientes podem ser alterados usando as barras deslizantes. A cada alteração, um novo gráfico é imediatamente gerado, tornando o ambiente virtual mais dinâmico para o estudante, com mais interatividade e oportunidade para reflexão das consequências das escolhas dos parâmetros. 
Na função linear o coeficiente a é a inclinação da reta, indicando se a função é crescente $(\mathrm{a}>0)$ ou decrescente $(\mathrm{a}<0)$, e b é o intercepto da função com o eixo y. Para a $=0$ tem-se a função constante $\mathrm{f}(\mathrm{x})=\mathrm{b}$. No aplicativo ilustrado na Figura 1 , têm-se duas barras deslizantes, uma para cada parâmetro da função. Note que ao selecionar $\mathrm{a}=2 \mathrm{e} \mathrm{b}=2$, o usuário visualiza uma função linear crescente no lado direito do painel. Ao escolher um valor negativo para a, o aplicativo mostra, imediatamente, uma função linear decrescente. A visualização dessas duas situações e a possibilidade de escolher outros valores para a e b permite que o usuário compreenda melhor o efeito desses coeficientes.

Em uma função quadrática, o coeficiente a está associado à concavidade, b à inclinação, e c ao intercepto no eixo y. A seleção por essa função é feita pelo primeiro botão à esquerda do painel. Essa escolha resulta em três barras deslizantes, uma para cada coeficiente (a, b e c). Na Figura 2 ilustramos o aplicativo para a função em que $\mathrm{a}=2$ (concavidade voltada para cima), $\mathrm{b}=-4$ (parábola deslocada para a direita) e c $=1$.

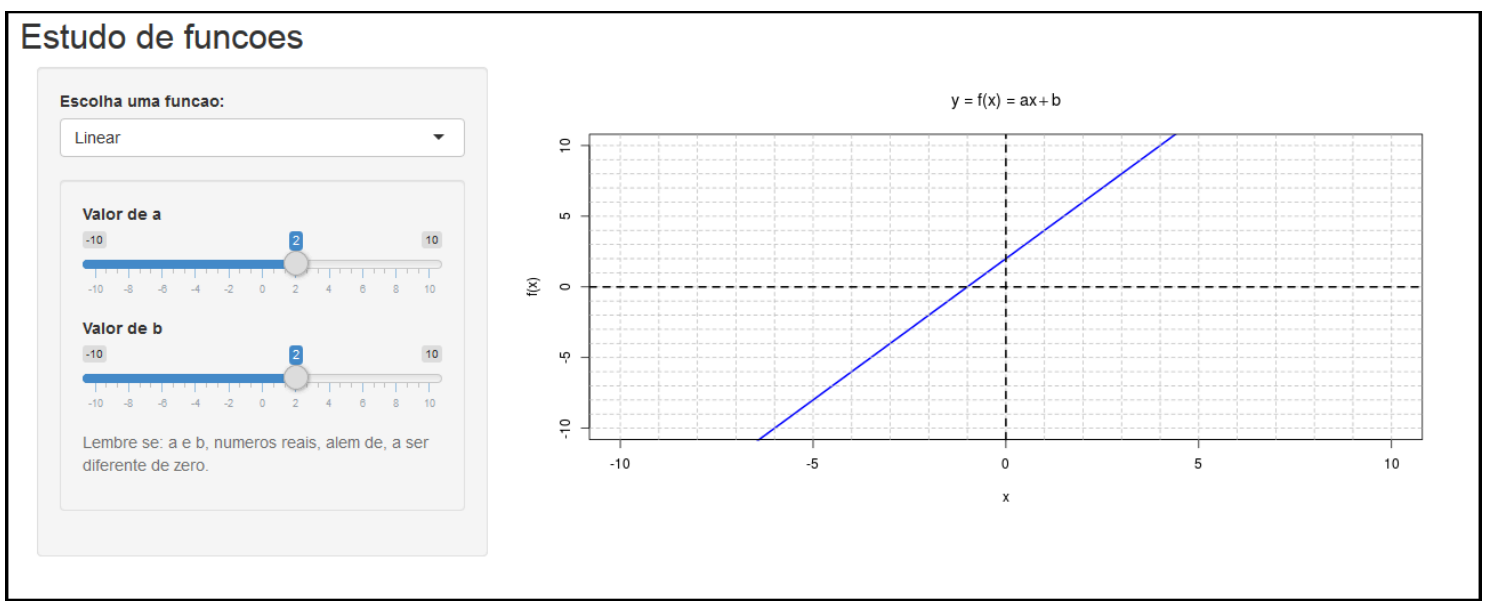

Figura 1: Ilustração do uso do aplicativo para o estudo de funções lineares com a $>0$.

Ao trabalhar com a função exponencial, o usuário poderá alterar os valores da base na barra deslizante. Já na composição função modular ele será capaz de visualizar que a constante escolhida determina se o gráfico é voltado para cima ou para baixo. Finalmente, ao usar o aplicativo para o estudo da função logarítmica ele poderá alterar o valor da base e verificar o seu efeito.

Para todas as funções desse aplicativo, abaixo da última barra deslizante, o usuário encontra um pequeno lembrete com os valores que podem ser utilizados para cada coeficiente, conforme descrito na Tabela 1. Além disso, a cor da linha do gráfico também pode ser alterada, contribuindo para a ludicidade e também para interpretação matemática do gráfico. 


\begin{tabular}{|c|c|c|}
\hline Função & Forma Algébrica & Condições \\
\hline \hline Linear & $\mathrm{f}(\mathrm{x})=\mathrm{ax}+\mathrm{b}$ & $\mathrm{a}, \mathrm{b} \in \mathbb{R}, \mathrm{a} \neq 0$ \\
\hline Quadrática & $\mathrm{f}(\mathrm{x})=\mathrm{ax}^{2}+\mathrm{bx}+\mathrm{c}$ & $\mathrm{a}, \mathrm{b}$ e $\mathrm{c} \in \mathbb{R}, \mathrm{a} \neq 0$ \\
\hline Exponencial & $\mathrm{f}(\mathrm{x})=\mathrm{a}^{\mathrm{x}}$ & $\mathrm{a} \in \mathbb{R}, \mathrm{a}>0$ e $\mathrm{a} \neq 1$ \\
\hline Composição Modular & $\mathrm{f}(\mathrm{x})=\mathrm{a}|\mathrm{x}|$ & $\mathrm{a} \in \mathbb{R} \mathrm{e} \mathrm{a} \neq 0$ \\
\hline Logarítimica & $\mathrm{f}(\mathrm{x})=\log _{\mathrm{a}} \mathrm{x}$ & $\mathrm{a}>0, \mathrm{a} \neq 1$ \\
\hline
\end{tabular}

Tabela 1: Funções implementadas no aplicativo criado.

\section{Estudo de funcoes}
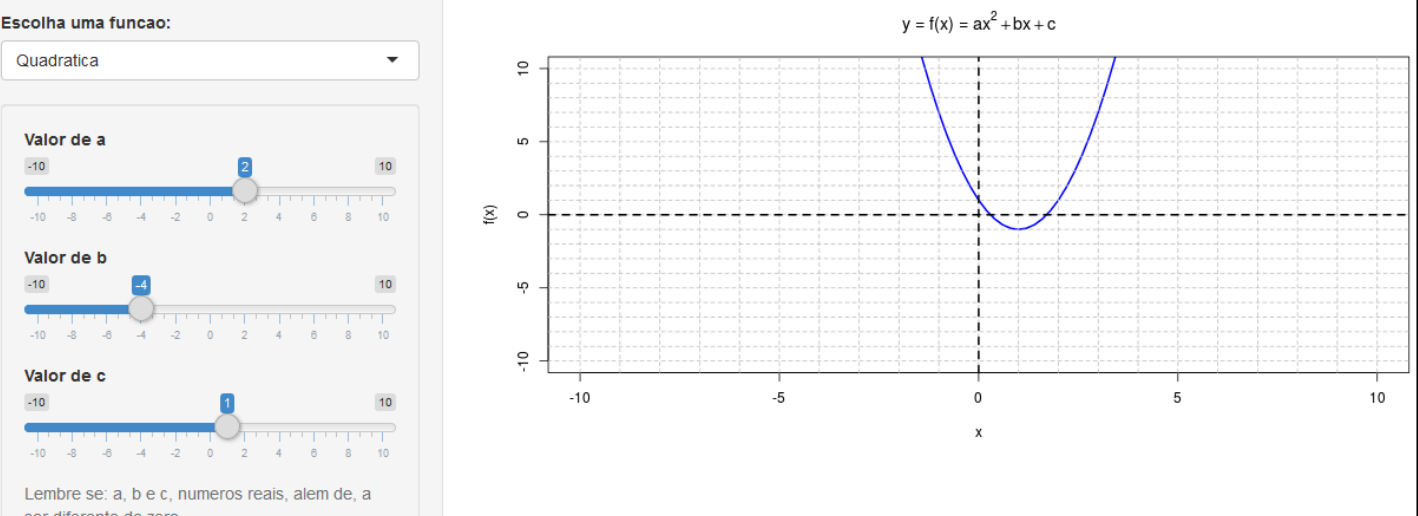

Figura 2: Ilustração do uso do aplicativo para o estudo de funções quadráticas com a $>0$, b $<0$ e $c>0$.

\subsection{Aplicativos para o ensino de Estatística Descritiva}

O conhecimento de Estatística é de grande importância em uma sociedade, principalmente diante da grande quantidade de informações veiculada diariamente por diversas mídias. Cidadãos comuns frequentemente usam informações de ordem estatística para fundamentar suas opiniões e tomadas de decisão. Então, apresentar a Estatística na educação básica é fundamental para que o estudante, ao receber uma informação estatística em seu cotidiano, saiba interpretá-la e esteja atento à maneira como ela foi obtida e como é divulgada [1].

De acordo com a BNCC (Base Nacional Comum Curricular [5]), a interpretação de dados em tabelas ou gráficos simples é iniciada partir do primeiro ano do ensino fundamental, evoluindo até o último ano do mesmo. O conceito de média é trabalhado a partir do $7^{\underline{0}}$ do ensino fundamental. Ainda no ensino fundamental, o conceito de mediana é tratado, inicialmente, já no $8^{\underline{0}}$ ano.

Nos aplicativos criados para o ensino de Estatística Descritiva são abordados gráficos para variáveis qualitativas e quantitativas, e os conceitos de valor mínimo, máximo, média e mediana. Ao iniciar ambos os aplicativos, o usuário visualizará, no lado direito do painel, uma mensagem de erro, escrita em vermelho, que é imediatamente substituída pelo gráfico assim que os dados são carregados. 


\subsection{Gráficos para variáveis qualitativas}

Uma variável qualitativa (categórica) é aquela cuja escala de medida consiste em um conjunto de categorias, como sexo (Masculino ou Feminino) e cor dos olhos (Azul, Castanho, Verde). Para um conjunto de dados, as frequências observadas nas categorias podem ser apresentadas em gráficos ou tabelas. O aplicativo criado auxilia no ensino do conceito de frequências e interpretação de gráficos de barras e de setores ( $p i z z a$ ). Ao utilizar esse aplicativo em sala de aula, o professor pode desenvolver atividades que envolvam a coleta de dados dos próprios estudantes, o que torna a aula ainda mais interativa e participativa.

Inicialmente o usuário carrega seu banco de dados, que é localizado pelo botão "Browse". Em seguida o usuário deve informar se o seu banco de dados contém um cabeçalho com o nome da variável. Então, pelos botões situados no lado esquerdo do painel, ele escolhe a variável que deseja visualizar e uma opção de gráfico (barras ou setores). Para o gráfico de barras também é possível alterar sua cor.

Nas Figuras 3 e 4 são apresentados os gráficos de barras e de setores, respectivamente, utilizando dados fictícios. Para a variável "cor de olhos" a categoria que ocorreu com maior frequência foi "castanho".

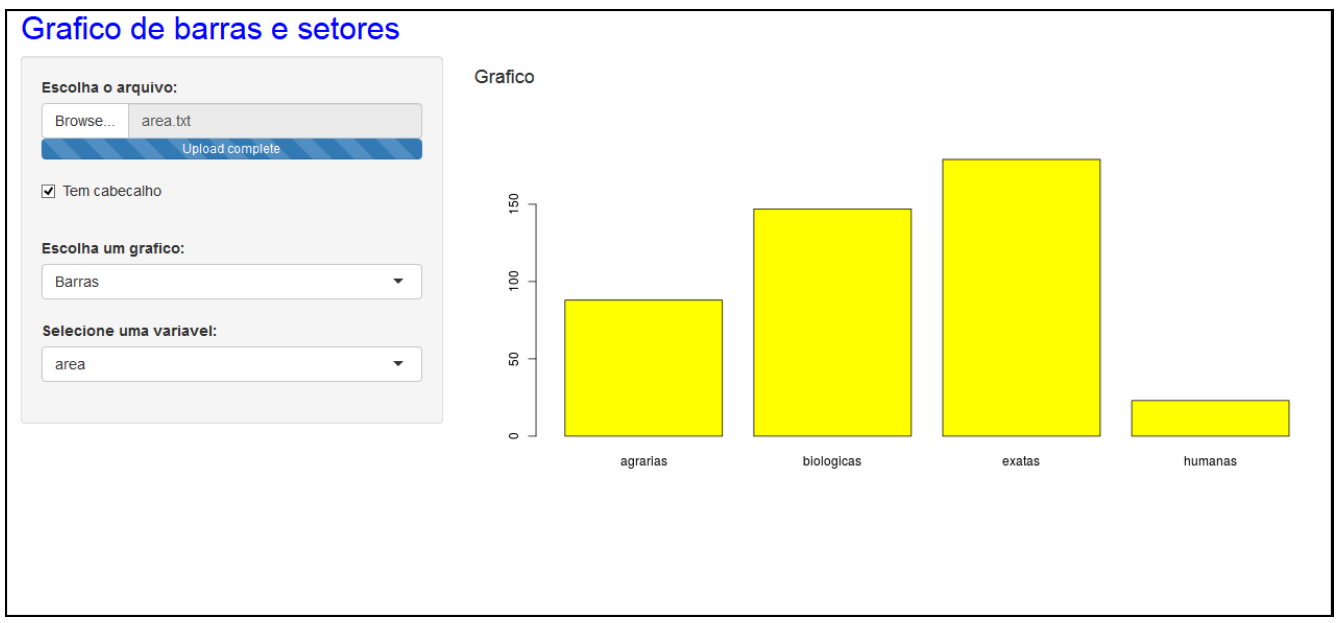

Figura 3: Ilustração do aplicativo para ensino de gráfico de barras. 


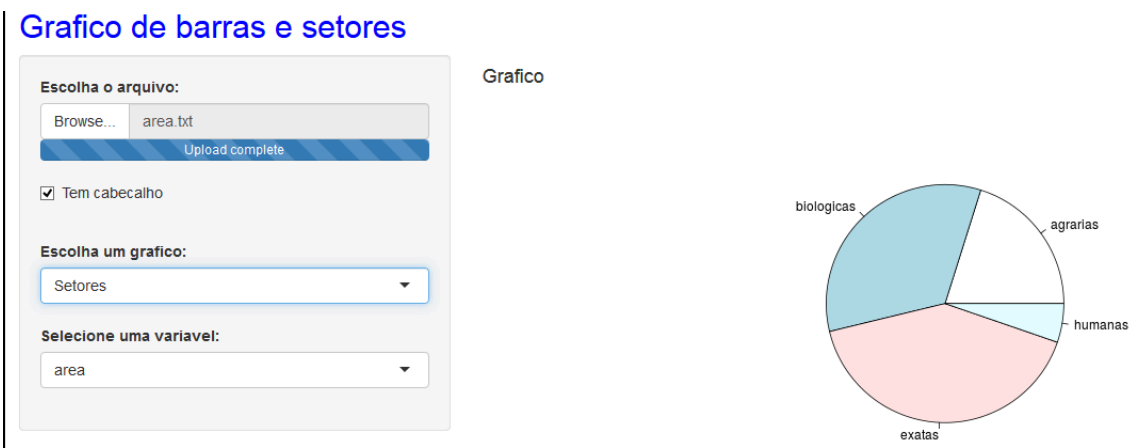

Figura 4: Ilustração do aplicativo para ensino de gráfico de setores.

\subsection{Gráficos e medidas estatísticas para variáveis quantitativas mensuradas}

Uma variável quantitativa contínua (mensurada) é aquela cujos dados são resultantes de um processo de mensuração, como peso, altura, renda e medidas antropométricas. No aplicativo criado é possível visualizar o gráfico (histograma) e medidas estatísticas (valor mínimo, máximo, média e mediana) de um conjunto de dados desse tipo.

Como sugerido na seção anterior, dados dos próprios estudantes podem ser utilizados. Para ilustrar as funcionalidades do aplicativo, utilizamos dados fictícios de altura de adultos (Figura 5). Por meio do gráfico gerado é possível trabalhar o conceito de frequência nas classes da variável, bem como noções de simetria e assimetria.

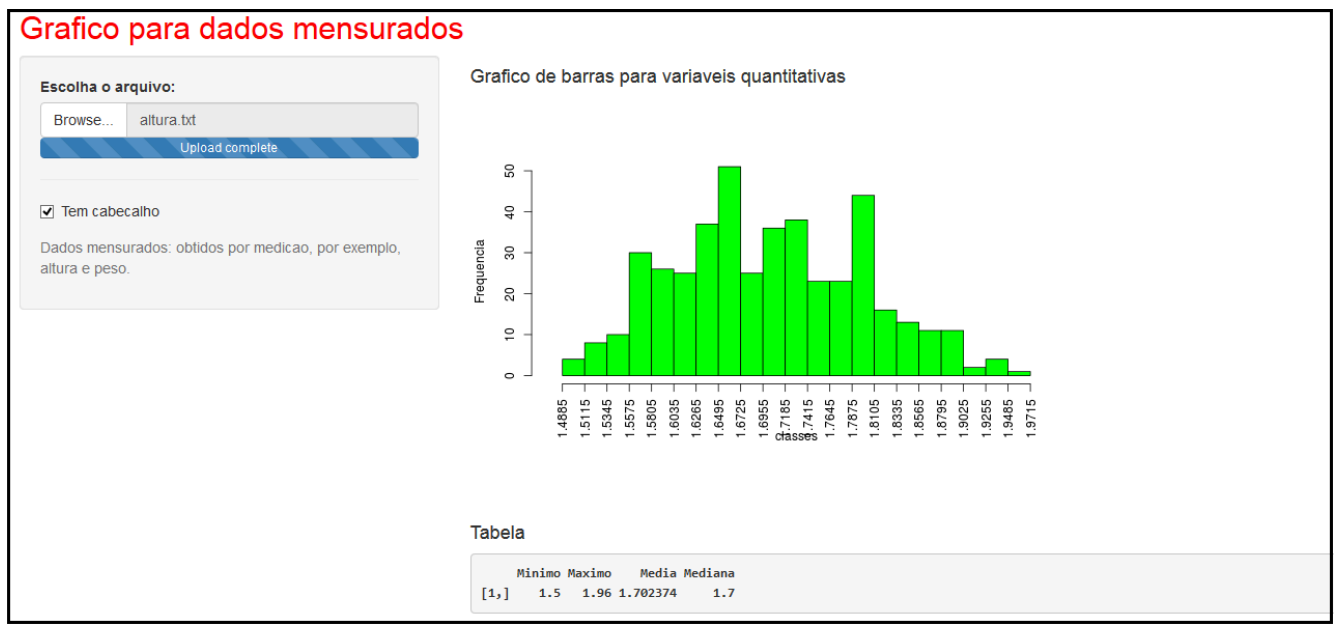

Figura 5: Ilustração do uso do aplicativo para gráfico e medidas estatísticas de variáveis (quantitativas) mensuradas. 


\section{Considerações Finais}

Diante do contexto da massificação dos recursos tecnológicos, torna-se necessário repensar as posturas, técnicas e recursos para o ensino básico como um todo. Computadores e dispositivos móveis sem fio fazem parte do dia a dia dos estudantes, e sua utilização pode potencializar, dinamizar e tornar mais lúdico o processo de ensino-aprendizagem. A aplicação dos recursos de Mobile Learning no ensino de matemática é uma área de pesquisa em plena expansão e que vem crescendo rapidamente. O uso de aplicativos em dispositivos móveis, pela alta aceitação entre os estudantes mais jovens e de todas as idades, pode expandir as fronteiras do ensino de matemática [4]. Este estudo teve como objetivo criar, implementar e disponibilizar três aplicativos para o ensino de conceitos de Matemática e Estatística na Educação Básica. O uso pedagógico desses conceitos não faz parte do escopo desse trabalho, mas pode ser avaliado em pesquisas futuras.

Para a disciplina de Matemática criou-se um aplicativo para o estudo de funções, dando autonomia para o estudante explorar as possíveis modificações nos parâmetros das funções e as consequências dessas modificações na interpretação gráfica dos resultados, permitindo a reflexão e a construção de ideias dos temas abordados. Nos aplicativos para o ensino de Estatística Descritiva o enfoque foi dado na interpretação de gráficos e nas medidas de máximo, mínimo, média e mediana de um conjunto de dados. A grande vantagem é que os dados a serem explorados podem ser coletados entre os próprios estudantes, promovendo um ambiente mais participativo.

A princípio, a necessidade de ter um dispositivo com conexão à internet para o acesso aos aplicativos pode ser um complicador no contexto atual das escolas de Ensino Fundamental e Médio. Então é importante ressaltar que o acesso por um navegador web é a maneira mais simples de compartilhar e utilizar os aplicativos, mas não é a única. Com os scripts ui.R e server.R, e um computador com o programa $\mathrm{R}$ instalado, o professor pode reproduzir todos os aplicativos criados, sem necessidade de internet. Além disso, apesar do pacote Shiny no R não ser tão popular no ensino de matemática como o GeoGebra, por exemplo, sua utilização permite que o usuário, seja ele estudante ou professor, possa avançar e aprofundar na interação com o R e o RStudio, tanto em termos de linguagem de programação avançada, quanto em termos de conteúdos estatísticos aprofundados.

Finalmente, uma das grandes vantagens de utilizar o Shiny na criação de aplicativos para o ensino é que o R é um programa gratuito e livre. Então é possível que o professor crie suas próprias rotinas, tendo autonomia para adaptar todo o conteúdo dos aplicativos de acordo com a realidade onde está inserido.

\section{Referências}

[1] Barberino, M. R. B , Magalhães, M. N. e Nacimento M. Uma proposta para desenvolver o letramento estatístico através de projetos.2015. Disponível em: <https://www.periodicos.unifra.br/ index.php/VIDYA>. Acesso em: 26 de agosto de 2021.

[2] Beeley, C. Web Application Development with $R$ Using Shiny. Birmingham: Packt, 2016.

[3] Biembengut, M. S. Modelagem Matemática \& Resolução de Problemas, Projetos e Etnomatemática: Pontos Confluentes. Alexandria Revista de Educação em Ciência e Tecnologia, v.7, n.2, p.197-219, 2014.

[4] Borba, M., Askar, P., Engelbrecht, J., Gadanidis, G., Linares, S. e Aguilar, M. S. lended learning, e-learning and mobile learning in mathematics education. ZDM Mathematics Education, v.48, p.589-610, 2016. 
[5] Brasil. Ministério da Educação.Base Nacional Comum Curricular.Brasília. 2018. Disponível em:<http://basenacionalcomum.mec.gov.br/a-base>. Acesso em: 26 de agosto de 2021.

[6] Carneiro, M. J. D., Spira, M., Sabatucci, J. Proposta Curricular de Matemática para o Ensino Fundamental e Ensino Médio. 2008.

[7] Chang, W., Cheng, J. Allaire, J. J., Xie, Y., Yihui, M. e Mcpherson, J. Shiny: Web Application Framework for $R$. $R$ package version 1.0.5. 2018. Disponível em: <https://CRAN.R-project.org/ package=shinyhttps://CRAN.R-project.org/package=shiny $>$. Acesso em: 30 de abril de 2018 .

[8] Doi, J., Potter, G., Wong, J., Alcaraz, I. and Chi, P. "Web Application Teaching Tools for Statistics Using R and Shiny". Journal of Technology Innovations in Statistics Education. Disponível em:<https://escholarship.org/uc/item/00d4q8cp>. Acesso em: 12 de novembro de 2020.

[9] Fawcett, L. "Using Interactive Shiny Applications to Facilitate Research-Informed Learning and Teaching". Journal of Statistics Education, v.26, n.1, p. 2-26, 2018.

[10] Freitas, R. O. e Carvalho, M. "Tecnologias móveis: tablets e smartphones no ensino da matemática". Laplage em Revista, Sorocaba, v.3, n.2, p.47-61, mai.-ago. 2017.

[11] Henning, E., Ramos, M. S., Aguiar, R., Siple, I. Z e Santos, , L. M. "Para além da computação estatística: o uso do ambiente R para o ensino de métodos numéricos". Revista Novas Tecnologias na Educação, Rio Grande do Sul, v.14, n.1, julho 2016.

[12] Konrath, A. C., Silva, S. A., Henning, E., Santos, L. M., Miranda, R. G. e Samohyl, R. W. "Desenvolvimento de Aplicativos Web Com R e Shiny: inovações no ensino de Estatística". Abakos, Belo Horizonte, v. 6, n. 2, p. 55-71, maio 2018.

[13] Muller, T. J. e Zabala, F. J. "Avaliação e correção automática no software livre RStudio". Revista Novas Tecnologias na Educação, Rio Grande do Sul, v.14, n.1, julho 2016.

[14] Reimer, K. e Moyer, P. S. "Third graders learn about fractions using virtual manipulatives: A classroom study". Journal of Computers in Mathematics and Science Teaching, Salt Lake City, v.24, n.1, p. 5-25, 2005.

[15] R Core Team. $R$ : A language and environment for statistical computing. $R$ Foundation for Statistical Computing. Vienna, Austria, 2018. Disponível em: <https://www.R-project.org/>. Acesso em: 12 de novembro de 2020 .

[16] Schreiber, K. P., Battisti, I. K. e Kolterman, I. "Processos de Compreensão pelo Professor em Formação Inicial ao Ensinar Função de $1^{\circ}$ grau Considerando o Uso de um Software". Alexandria Revista de Educação em Ciência e Tecnologia, Florianópolis, v.10, n.2, p.127-145, nov. 2017.

[17] Verzani, J. Getting started with RStudio. Published by O'Reilly. 2011.

[18] Williams, I. J. e Williams, K. K. "Using an R shiny to enhance learning experience of confidence intervals". Journal of Statistics Education, Hoboken, v.1, n.40, p. 24-28, 2017. 
Paula Ribeiro dos Santos Escola Superior de Agricultura Luiz de Queiroz, Universidade de São Paulo,

Piracicaba, SP, Brasil $<$ paullasant_s@hotmail.com>

Izabela Regina Cardoso de Oliveira Departamento de Estatística, Universidade Federal de Lavras, Lavras, MG, Brasil <izabela.oliveira@ufla.br>

Evelise Roman Corbalan Góis Freire Departamento de Matemática e Matemática Aplicada, Universidade Federal de Lavras, Lavras, MG, Brasil $<$ evelise.freire@ufla.br>

Recebido: 30/08/2021

Publicado: $17 / 02 / 2022$ 